\title{
DIVERSIDADE DE PEIXES NA ZONA COSTEIRA DO ESTADO DO MARANHÃO A PARTIR DE DADOS DA PESCA ESPORTIVA
}

\section{FISH DIVERSITY IN THE MARANHÃO STATE COASTAL ZONE FROM RECREATIONAL FISHERY DATA}

\author{
Jadson Pinheiro Santos ${ }^{1}$; Matheus Willy Machado Ferreira ${ }^{2}$; Kleiton Belfort Ferreira ${ }^{3}$; Lucas Douglas Barros \\ Fonseca ${ }^{4}$ Kaio Lopes de Lima ${ }^{5}$; Erick Cristofore Guimarães ${ }^{6} \&$ Danilo Francisco Corrêa Lopes \\ ${ }^{1,5}$ Departamento de Engenharia de Pesca, Universidade Estadual do Maranhão - Uema \\ ${ }_{1,2,3,4}$ Laboratório de Ictiofauna e Piscicultura Integrada, Universidade Estadual do Maranhão - Uema; \\ ${ }^{6}$ Programa de Pós-graduação Sociedade, Natureza e Desenvolvimento, Instituto de Ciências da Educação, \\ Universidade Federal do Oeste do Pará - Ufopa; \\ ${ }^{7}$ Coordenação de Ciências do Mar, Universidade Federal do Maranhão - Ufma
}

*e-mail: jadsonsantos@professor.uema.br

Recebido em 05 de abril de 2021- Publicado em 07 de junho de 2021

RESUMO O estado do Maranhão, possui um vasto litoral que propicia o desenvolvimento da pesca esportiva litorânea, atividade que tem atraído cada vez mais adeptos nos últimos anos. Neste contexto, o objetivo deste trabalho foi demonstrar a importância da pesca esportiva como fonte auxiliar no acompanhamento da diversidade de peixes nas praias da região metropolitana de São Luís, Maranhão, através do acompanhamento dos torneios de pesca esportiva, realizados em dezembro de 2018 na praia do Olho d'Água e em julho de 2019, na praia do Araçagi. Os peixes capturados foram acondicionados em caixas isotérmicas contendo gelo e transportados ao laboratório para identificação taxonômica. Foram capturados 252 espécimes, pertencentes a 19 espécies e distribuídos em 10 famílias. A família Sciaenidae foi a mais representativa com 3 espécies. As espécies predominantes foram Conodon nobilis e Geniatremus luteus da família Haemulidae, que juntas representaram $39 \%$ dos espécimes capturados na praia do Olho d'Água e $24 \%$ na praia do Araçagi. Os indivíduos de C. nobilis apresentaram peso médio de 29,4 $\pm 12,7 \mathrm{~g}$ e comprimento médio $12,3 \pm 2,0 \mathrm{~cm}$, com curva de relação peso $x$ comprimento representada pela equação $\mathrm{PT}=0,034 \mathrm{CT}^{2,6637}$ e $\mathrm{R}^{2}=0,67$, com $\mathrm{b}=2,67$. Enquanto em $G$. luteus, os indivíduos apresentaram peso médio de $96,5 \pm 82,2$ g e comprimento médio de $15,8 \pm 4,4 \mathrm{~cm}$, com curva da relação peso $\mathrm{x}$ comprimento representado pela equação: $\mathrm{PT}=0,0145 \mathrm{CT}^{3,0999}$ e $\mathrm{R}^{2}=0,81$, com $\mathrm{b}=$ 3,09. A diversidade de peixes em pescarias de praia, pode contribuir com estudos de biologia pesqueira de modo a subsidiar o desenvolvimento de estratégias de manejo e conservação dos estoques pesqueiros.

Palavras-chave: costa amazônica; ictiofauna; biologia pesqueira; recursos pesqueiros.
ABSTRACT The state of Maranhão has a vast coastline that promotes the development of coastal recreational fishery, an activity that has attracted more and more fans in recent years. In this context, the objective of this work was to demonstrate the importance of sport fishing as an auxiliary source in monitoring the diversity of fish on the beaches of the metropolitan region of São Luís, Maranhão, through the monitoring of sport fishing tournaments, held in December 2018 on the beach do Olho d'água and in July 2019, at Araçagi beach. The captured fish were stored in isothermal boxes containing ice and transported to the laboratory for taxonomic identification. 252 specimens were captured, belonging to 19 species, and distributed in 10 families. The family Sciaenidae was the most representative with 3 species. The predominant species were Conodon nobilis and Genyatremus luteus from the Haemulidae family, which together represented $39 \%$ of the specimens captured on the Olho d'água beach and $24 \%$ on the Araçagi beach. The individuals of $\mathrm{C}$. nobilis had an average weight of $29.4 \pm 12.7 \mathrm{~g}$ and an average length of $12.3 \pm 2.0 \mathrm{~cm}$, with a curve of weight $x$ length ratio represented by the equation $\mathrm{PT}=0.034 \mathrm{CT}^{2.6637}$ and $\mathrm{R} 2=0,67$, with $\mathrm{b}=$ 2.67. While in G. luteus, the individuals had an average weight of $96.5 \pm 82.2 \mathrm{~g}$ and an average length of $15.8 \pm$ $4.4 \mathrm{~cm}$, with a curve of the weight $\mathrm{x}$ length ratio represented by the equation: $\mathrm{PT}=0.0145 \mathrm{CT}^{3.0999}$ and $\mathrm{R} 2$ $=0.81$, with $\mathrm{b}=3.09$. The diversity of fish in beach fisheries can contribute to studies of fishery biology to support the development of management strategies and conservation of fish stocks.

Key words: Amazon coast; ichthyofauna; fisheries biology; fishery resources. 


\section{Introdução}

A pesca é uma das atividades produtivas mais antigas da humanidade onde os recursos pesqueiros marítimos, costeiros e continentais tornaram-se importante fonte de renda, geração de trabalho e alimento e tem contribuído para a permanência do homem no seu local de origem (Rodrigues, 2013). Com o passar do tempo e a partir da concepção da lei no 11.959 , de 29 de junho de 2009, conhecida como a "Lei da Pesca" no Brasil, a atividade pesqueira foi dividida em: pesca comercial e não comercial, propondo ainda a definição de pesca amadora como uma atividade de pesca praticada por brasileiro ou estrangeiro, com uso de equipamentos ou petrechos previsto em legislação específica, tendo por finalidade o lazer ou desporto (Brasil, 2009).

A atividade da pesca esportiva tem apresentado um crescimento significativo no Brasil favorecida pela biodiversidade de peixes e variedade de ambientes encontrados no país (Albano \& Vasconcelos, 2013), além de apresentar forte correlação ao turismo de pesca. Os principais produtos economicamente valorados da pesca esportiva associados ao turismo incluem os bens e serviços que os pescadores esportivos compram para a prática, a saber: equipamentos de pesca, transporte, hospedagem, alimentação e serviços especializados como aluguel de barco e equipamentos e a contratação de guias de pesca (Catella, 2003). O turismo de pesca proporciona grandes emoções ao praticante, mas também é uma maneira de garantir o emprego de muitas pessoas que dependem da pesca amadora como fonte de emprego e renda, principalmente para as populações locais (Brasil, 2010).

A criação da portaria $\mathrm{n}^{\circ}$ 91, de 4 de fevereiro de 2020, que autoriza a pesca esportiva em unidades de conservação ambiental, trouxe à pesca esportiva o entendimento de ser uma atividade reconhecida como um dos tipos de pesca amadora, praticada em modalidade pesque e solte, na qual o peixe é devolvido vivo ao seu habitat (ICMBIO, 2020). Ainda de acordo com a portaria, uma das condições para a autorização da pesca esportiva é que os planejamentos das atividades levem em conta a necessidade de os recursos naturais serem usados de forma racional e sustentável (ICMBIO, 2020).

O estado do Maranhão, possui o segundo maior litoral do Brasil propiciando o desenvolvimento da atividade de pesca esportiva, principalmente nas praias da região costeira, além de ter a prática favorecida pelo fato de parte do estado estar inserido geograficamente em uma área transicional sob a influência de três grandes domínios biogeográficos, que são: bioma Amazônico, caatinga e cerrado, permitindo inferir que o Maranhão aparece como uma das áreas brasileiras de maior diversidade animal e vegetal (Cardoso et al., 2018).

Apesar do grande potencial, vale ressaltar que a escassez de informações sobre os estoques pesqueiros vem de encontro à falta de recursos para a condução de estudos que realizem levantamentos regulares, de forma que que os dados populacionais sejam atualizados permitindo a elaboração de ações de gestão para regulação e controle da exploração racional dos estoques pesqueiros. No relatório The State of World Fisheries and Aquaculture (FAO, 2020), o Brasil foi mencionado por não ter disponibilizado dados oficiais da produção pesqueira desde 2014 (Filho, 2020). Para a manutenção da pesca como uma atividade viável no futuro é necessário que ocorra a gestão sustentável do setor, com atenção a conservação das espécies e aos ecossistemas aquáticos, com amplo significado econômico e social para as localidades e países onde ela é praticada (Pitcher, 1999).

$\mathrm{Na}$ tentativa de suprir a necessidade de informações mais atuais e precisas da diversidade de peixes em determinadas regiões, a pesca amadora apresenta-se como uma alternativa para contribuir com a geração de informações a serem utilizadas por órgãos governamentais e não governamentais na atualização dos dados e desenvolvimento de ações mais efetivas para a conservação da biodiversidade de peixes em toda a costa brasileira. Neste contexto, o presente trabalho teve como objetivo demonstrar a importância da pesca esportiva no acompanhamento da diversidade de peixes nas praias da região metropolitana de São Luís, Estado do Maranhão.

\section{Material e Métodos}

\section{ÁREA DE ESTUDO}

O trabalho foi realizado no período de 2018 e 2019 nas praias do Olho d'Água localizada no município de São Luís, entre as coordenadas $2^{\circ} 28^{\prime} 43.25^{\prime}$ 'S e $44^{\circ} 13^{\prime} 45.69^{\prime \prime} \mathrm{W}$, e na praia do Araçagi, situada no município de Paço do Lumiar, entre as coordenadas de $2^{\circ} 27^{\prime} 53.65^{\prime}$ 'S e 44 11' 50.99' 'W (Figura 1). 


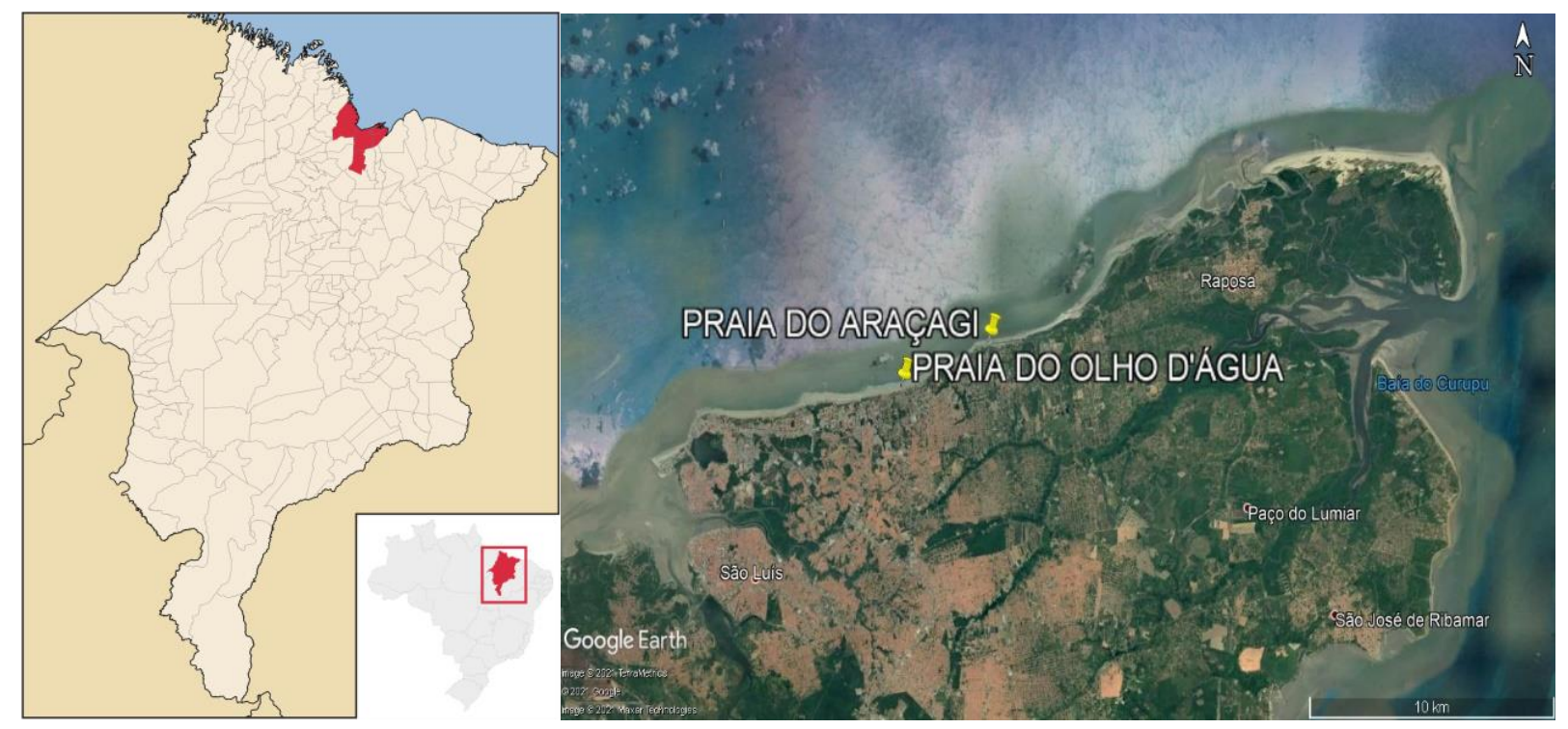

Figura 1- Mapa de localização das áreas de estudos: Praias do Olho d'Água e do Araçagi, Estado do Maranhão, Brasil. (Fonte: adaptado do Google Earth Pro).

\section{COLETA DE DADOS}

Foram realizadas observações a partir das espécies capturadas pelos competidores. Durante o torneio, os pescadores utilizaram vara, molinete, linha, anzol e chicote, além de iscas naturais, como camarão cinza Litopenaeus vannamei (Boone, 1931), camarão piticaia Xiphopenaeus kroyeri (Heller, 1862) e o corrupto Callichirus major (Stimpson, 1866).

Nos registros, foram anotados: o ponto de coleta, hora, nome vulgar e a identificação taxonômica até a menor categoria possível. Quando não foi possível identificar os peixes capturados a nível de espécie, os respectivos exemplares foram encaminhados para o Laboratório de Ictiofauna e Piscicultura Integrada da Universidade Estadual do Maranhão (LABIPI), vinculado ao curso de Engenharia de Pesca da Universidade Estadual do Maranhão para posterior identificação.

Os peixes foram fixados em formalina $10 \%$, permanecendo por aproximadamente 10 dias nesta solução e posteriormente conservados em uma solução de álcool etílico a $70 \%$. A triagem e identificação taxonômica foi feita até a menor categoria, quando possível, com base em bibliografia especializada, coleções de referência ou banco de imagens. A classificação taxonômica, os nomes de espécies considerados como válidos, autores e anos das descrições das espécies, e distribuição geográfica, se basearam nas compilações propostas por Fricke et al. (2021a,b), onde os autores reúnem todas as principais e mais recentes classificações para cada grupo de peixe. Os dados foram tabulados em software de edição de planilha para se obter a diversidade relativa de espécies de peixes capturados.

\section{ANÁLISE ESTATÍSTICA}

Nas competições de pesca de praia realizadas na região metropolitana de ilha de São Luís foram registrados dados do peso e comprimento das espécies C. nobilis e G.luteus, como forma de se obter dados biológicos para o acompanhamento dos estoques pesqueiros. $\mathrm{O}$ cálculo da relação peso x comprimento dos dois peixes foi realizado a partir da aferição do comprimento total, expresso em centímetros $(\mathrm{cm}) \mathrm{com} o$ auxílio de um ictiômetro, e do peso total, expresso em gramas $(\mathrm{g})$ com auxílio de uma balança com precisão de 3 casas decimais. A relação peso - comprimento além de fornecer parâmetros que permitem a avaliação do índice de bem-estar das populações de peixes (Vazzoler, 1996) permite também estimativas do peso médio dos peixes uma vez conhecido o comprimento (Beyer, 1987).

Após os dados tabulados, foi aplicada a equação: PT=a.CT ${ }^{\mathrm{b}}$ (Ivo \& Fontelles-Filho, 1997; Zar, 2010), em que "PT" corresponde ao peso total, "CT", ao comprimento total, "a", ao fator relacionado pelo grau de 
engorda dos indivíduos e "b", ao coeficiente de alometria relacionado com o crescimento dos indivíduos. Concomitantemente, foram determinadas as alometrias das relações considerando o parâmetro 'b' (modelo linear: $b<1=$ alométrico negativo, $b=1=$ isométrico, $b>1=$ alométrico positivo; modelo geométrico: $\mathrm{b}<3=$ alométrico negativo, $\mathrm{b}=3=$ =isométrico, $\mathrm{b}>3=$ alométrico positivo) (Fonteles-Filho, 2011).

\section{Resultados e Discussão}

Nos torneios realizados na praia do Olho d'Água e Aracagy foram triados e identificados 252 espécimes pertencentes, a 19 espécies e 10 famílias (Tabela 1). As famílias que apresentaram maior representatividade no total de espécies capturadas foram Scianidae (34\%), Haemulidae (31\%) e Carangidae (16\%) (Figura 2).

Tabela 1. Relação das famílias, espécies, nome vulgar e área de captura dos peixes capturados nos torneios de pesca de praia na ilha de São Luís, Estado do Maranhão (1 Olho D’àgua - 2 Araçagi).

\begin{tabular}{|c|c|c|c|c|}
\hline \multirow{2}{*}{ Família } & \multirow{2}{*}{ Nome Científico } & \multirow{2}{*}{ Nome Vulgar } & \multicolumn{2}{|c|}{ Local } \\
\hline & & & 1 & 2 \\
\hline Polynemidae & Polydactylus virginicus (Linnaeus, 1758) & Barbudo & $\mathrm{X}$ & $\mathrm{X}$ \\
\hline Gerreidae & Diapterus rhombeus (Cuvier, 1829) & Carapeba & $\mathrm{X}$ & - \\
\hline \multirow[t]{4}{*}{ Carangidae } & Caranx hippos (Linnaeus, 1766) & Xaréu & $\mathrm{X}$ & $\mathrm{X}$ \\
\hline & Oligoplites palometa (Cuvier 1832) & Timbiro & $\mathrm{X}$ & $\mathrm{X}$ \\
\hline & Trachinotus cf. goodie (Jordan \& Evermann, 1896) & Pampo-Galhudo & $\mathrm{X}$ & - \\
\hline & Trachinotus falcatus (Linnaeus, 1758) & Pampo & $\mathrm{X}$ & $\mathrm{X}$ \\
\hline Tetraodontidae & Celomesus psittacus (Bloch \& Schneider, 1801) & Baiacu & $\mathrm{X}$ & $\mathrm{X}$ \\
\hline \multirow[t]{2}{*}{ Haemulidae } & Geniatremus luteus (Bloch, 1790) & Peixe-pedra & $\mathrm{X}$ & $\mathrm{X}$ \\
\hline & Conodon nobilis (Linnaeus, 1758) & Jiquiri & $\mathrm{X}$ & $\mathrm{X}$ \\
\hline \multirow[t]{4}{*}{ Ariidae } & Arius proops (Valenciennes, 1840) & Uritinga & $\mathrm{X}$ & $\mathrm{X}$ \\
\hline & Bagre bagre (Linnaeus, 1766) & Banderado & $\mathrm{X}$ & - \\
\hline & Cathorops spixii (Agassiz, 1829) & Bagre-amarelo & $\mathrm{X}$ & - \\
\hline & Aspistor quadriscutis(Valenciennes, 1840) & Cangatã & $\mathrm{X}$ & - \\
\hline \multirow[t]{3}{*}{ Sciaenidae } & Micropogonias furnieri (Desmarest, 1823) & Corvina & $\mathrm{X}$ & $\mathrm{X}$ \\
\hline & Menticirrhus americanos (Linnaeus, 1758) & Papa-terra & $\mathrm{X}$ & $\mathrm{X}$ \\
\hline & Macrodon ancylodon (Bloch \& Schneider, 1801) & Pescadinha-gó & - & $\mathrm{X}$ \\
\hline Batrachoididae & Batrachoides surinamensis (Bloch \& Schneider 1801) & Pacamão & - & $\mathrm{X}$ \\
\hline Elopidae & Elops saurus (Linnaeus, 1766) & Ubarana & - & $\mathrm{X}$ \\
\hline Echeneidae & Remora osteochir (Cuvier, 1829) & Remora & - & $\mathrm{X}$ \\
\hline
\end{tabular}

As espécies pertencentes à família Sciaenidae com maior representatividade foram Menticirrhus americanos, Micropogonias furnieri, e Macrodon ancylodon. Esta família compreende 69 gêneros com 293 espécies (Fricke et al., 2021b), sendo um dos relevantes recursos pesqueiros para a pesca comercial no Brasil e, principalmente, no estado do Maranhão. A família Sciaenidae é considerada parte integrante e predominante da comunidade de peixes demersais da plataforma continental brasileira (Vazzoler, 1991). As espécies dessa família habitam ambientes de água doce, estuarinos e marinho costeiro, incluindo Oceanos Atlântico, Indico e Pacífico (Zagonel, 2006).

Menticirrhus americanus é conhecida como papa-terra ou betara habitando fundos de areia ou de areia e lama, em águas costeiras de pouca profundidade e em regiões estuarinas (Rondineli et al., 2007). Distribuise desde a costa leste do Estados Unidos até Buenos Aires (Argentina) (Menezes \& Figueiredo, 1980). Pode alcançar até $50 \mathrm{~cm}$ de comprimento e a longevidade estimada em 10 anos (Castillo, 1986). No sul do Brasil, as betaras são importantes para a pesca comercial e esportiva, procuradas principalmente por pescadores de caniço (Lewis et al., 2001; Haluch, 2008; Basaglia \& Vieira, 2005), além de constituírem parte importante da comunidade de peixes demersais nas águas costeiras das regiões sul e sudeste do Brasil, tendo consequentemente, um importante papel na cadeia alimentar da comunidade (Vazzoller, 1975; Haimovici et al, 1996; Haluch, 2008). 


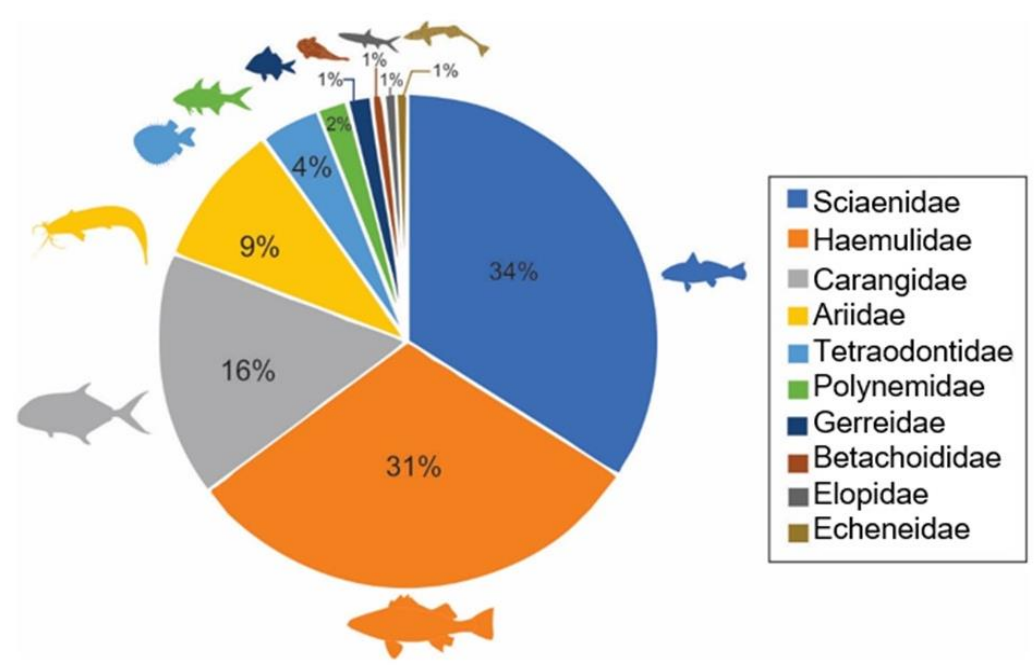

Figura 2. Principais famílias capturadas nos torneios de pesca nas praias do Olho d’Água e Araçagi, São Luís, Estado do Maranhão.

Micropogonias furnieri, popularmente conhecida por corvina, é uma espécie comum na atividade pesqueira. Apresenta ampla distribuição geográfica, ocorrendo das Antilhas e América Central (Costa Rica) até a Argentina (Menezes \& Figueiredo, 1980). Essa espécie demersal costeira, desova em locais próximos a baias e estuários para onde os indivíduos são recrutados e encontram maior disponibilidade de alimento e maior proteção durante o desenvolvimento inicial da vida (Vazzoler, 1991). É uma espécie costeira encontrada em profundidade de até $60 \mathrm{~m}$, com hábito alimentar caracterizado como carnívoro/detritívoro, pois na fase juvenil alimentam-se de invertebrados bentônicos e na fase adulta de pequenos peixes, vermes e crustáceos (Santana et al., 2016).

No Brasil, a corvina apresenta maiores densidades na região sudeste e sul (Magro et al., 2000; Mulato et al., 2015), sendo mais bem representada nos estuários subtropicais do que nos tropicais (Vilar et al., 2013; Mulato et al., 2015). Quanto aos desembarques comerciais, a corvina é o principal recurso demersal da frota pesqueira no Brasil, corresponde à segunda espécie mais capturada nos últimos anos, ficando atrás apenas da sardinha (MPA, 2010; Mulato et al., 2015).

Macrodon ancylodon é um cienídeo demersal marinho com ampla distribuição geográfica, ocorrendo desde as águas tropicais da Venezuela até as águas subtropicais da Argentina (Menezes \& Figueiredo, 1980). A espécie $M$. ancylodon conhecida como pescadinha-gó é frequentemente capturada ao longo de toda região estuarina no Maranhão e Pará, especialmente pelas comunidades da Raposa-MA e de Mosqueiro-PA (Santos, 2007). M. ancylodon é considerada uma espécie de grande importância econômica na pesca artesanal do estado do Maranhão (Figueiredo et al., 2014).

As espécies mais representativas da família Haemulidae, foram Genyatremus luteus (peixe-pedra) e Conodon nobilis (jiquiri). A família Haemulidae, pertence à ordem Perciformes *sedis mutabilis* e inclui 21 gêneros e 134 espécies (Fricke et al., 2021b). Em geral está família Haemulidae é composta por peixes de pequeno porte que atingem cerca de $50 \mathrm{~cm}$ (Cervigón et al., 1992), como o peixe-pedra G.luteus, que possui ocorrência restrita ao Atlântico ocidental desde o Sul do Caribe ao Brasil (Cervigón et al., 1992). A comercialização desta espécie ocorre principalmente nos estados do Maranhão e Pará (Almeida et al., 2011; Fernandes et al., 2015). É uma espécie bem aceita pela população maranhense, sendo um peixe de alto valor comercial sempre encontrado em feiras livres, supermercados, bares e restaurantes.

As espécies pertencentes à família Carangidae mais representativas foram: pampo-galhudo (Trachinotus goodei), xaréu (Caranx hippos), timbiro (Oligoplites palometa) e pampo (Trachinotus falcatus). No Brasil, a família Carangidae, é composta por 35 espécies, é tipicamente marinha e ocorre no Oceano Atlântico (Menezes et al., 2003). Possui importante valor comercial, sendo algumas espécies, alvo de pesca esportiva (Souza, 2016). Os Carangidae são pelágicos e formam cardumes (excetuando Alectis); algumas espécies podem estar relacionadas a recifes de corais, ou ainda penetrar em rios, mas ocorrem principalmente em ambientes estuarinos (Menezes \& Figueiredo, 1980). São frequentes também em baias e na zona de arrebentação, a qual é definida pela área de quebra de ondas na praia, sendo considerada uma região muito 
dinâmica, pois recebe influências constantes de ondas, marés e correntes que podem alterar sua topografia (Mclachlan, 1980). Este ambiente é de extrema importância para comunidade ictica, como área de refúgio, proteção e abundância de alimento, principalmente para os juvenis, propiciando condições adequadas ao crescimento (Felix et al., 2007).

Durante as análises foram observados para o $C$. nobilis peso médio de $29,4 \pm 12,7 \mathrm{~g}$ e comprimento médio $12,3 \pm 2,0 \mathrm{~cm}$ (Figura 3). A curva da relação peso $\mathrm{x}$ comprimento é representada pela equação: $\mathrm{PT}=$ $0,034 \mathrm{CT}^{2,6637}$ e $\mathrm{R}^{2}=0,67, \mathrm{com} \mathrm{b}=2,67$, demostrando que os exemplares de Jiquiri capturados apresentaram crescimento alométrico negativo, com peso aumentando mais lentamente do que o comprimento.

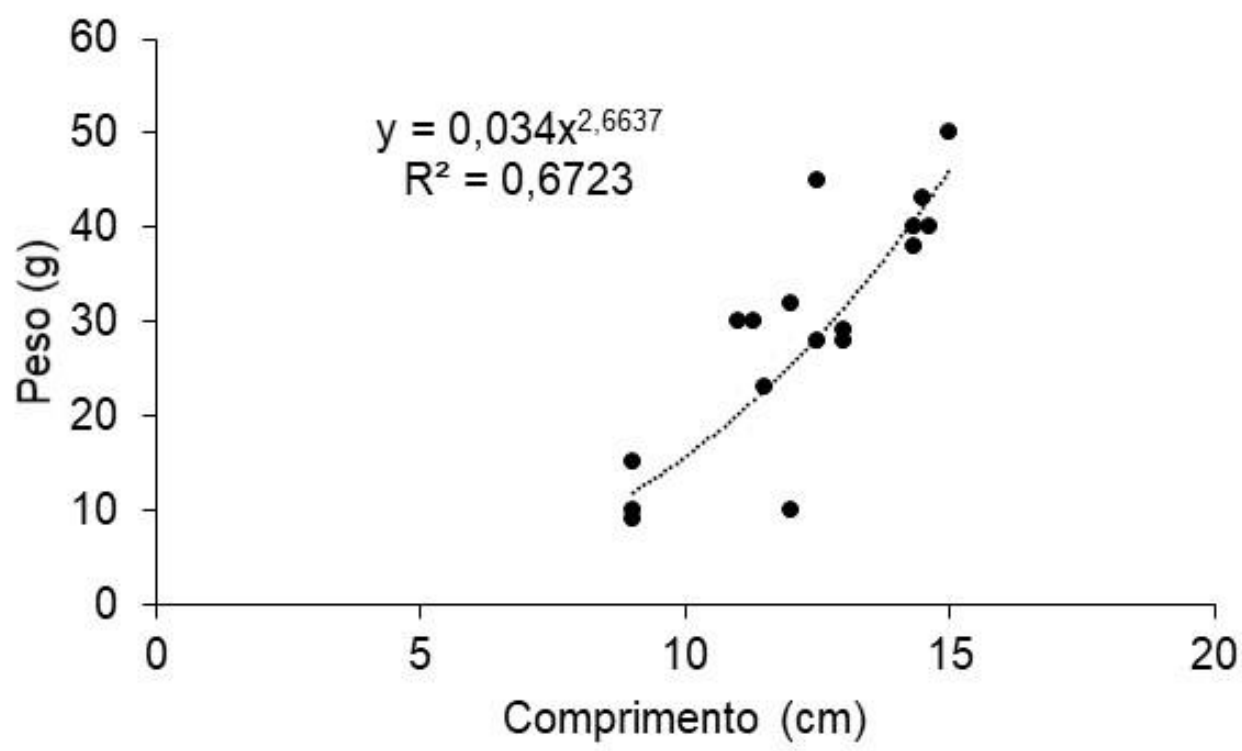

Figura 3. Variação peso x comprimento da espécie Conodon nobilis capturados em praias da região metropolitana de São Luís, Estado do Maranhão.

Para a espécie G. luteus, os exemplares capturados nos torneios apresentaram peso médio de $96,5 \pm 82,2$ g e comprimento médio de $15,8+4,4 \mathrm{~cm}$ (Figura 4). A curva da relação peso x comprimento é representada pela equação $\mathrm{PT}=0,0145 \mathrm{CT}^{3,0999}$ e $\mathrm{R}^{2}=0,81 \mathrm{com} b=3,09$, demostrando que os exemplares de peixe-pedra apresentaram crescimento alométrico positivo com peso aumentando mais rapidamente do que o comprimento.

As espécies $C$. nobilis e G. luteus são bastante frequentes nas pescarias esportivas e comerciais na região metropolitana de São Luís, G. luteus possui valor comercial e o $C$. nobilis possui pouco valor comercial (Santana et al., 2019). De acordo com Nunes et al. (2020), ao caracterizarem os tamanhos de primeira maturação de peixes com importância econômica na costa maranhense, estimam em $14,40 \mathrm{~cm}$ o de primeira maturação para o G. luteus, inferior ao comprimento médio encontrado no presente estudo.

Conodon nobilis é muito comum em todo litoral brasileiro, com ampla ocorrência na costa leste da Florida e do Texas (EUA), atinge pelo menos 32,0 cm de comprimento total. Os peixes dessa espécie são encontrados geralmente sobre fundos de pedra e de substrato mole, principalmente areia, com profundidades alcançando os 100 metros, se concentrando tipicamente em águas túrbidas, rasas e apresenta pouco valor comercial (Lopes et al., 2010).

Os exemplares analisados de $C$. nobilis apresentaram comprimento médio inferior ao encontrado por Lira et al. (2019), que estimou o comprimento de primeira maturação em 14,3 cm na costa de Pernambuco. Segundo os mesmos autores, esta espécie é frequentemente capturada como fauna acompanhante das pescarias de camarão e apresentam grande importância como elo da cadeia alimentar costeira. Nesse caso, apesar de ser imprescindível a obtenção de informações mais específicas sobre dieta, estrutura populacional e maturação sexual (Silva-Júnior et al., 2019), a pesca esportiva surge como fonte auxiliar para obtenção de informações sobre a diversidade de espécies ao longo do ano e monitoramento do tamanho e do peso dos espécimes capturados, possibilitando as estimativas da maturação do estoque das espécies. 


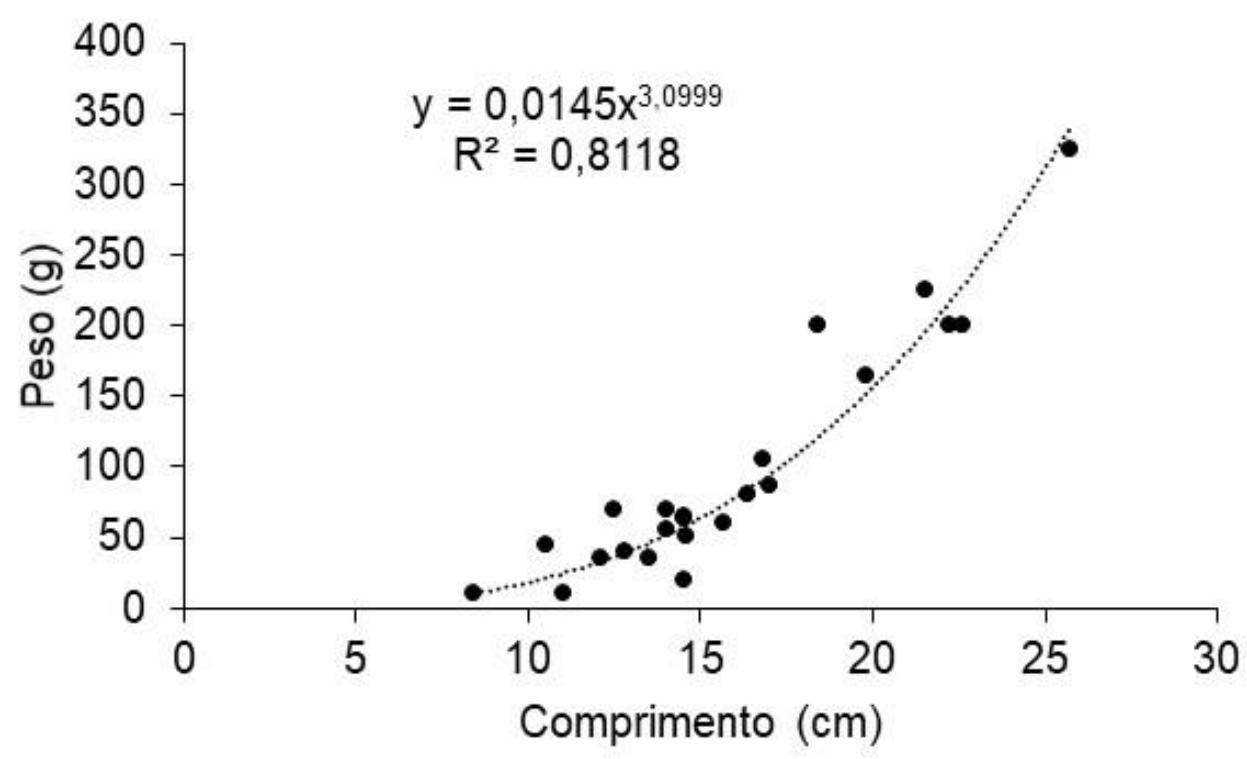

Figura 4. Variação de peso x comprimento da espécie Genyatremus luteus capturados em praias da região metropolitana de São Luís, Estado do Maranhão.

Os torneios de pesca vêm se tornando cada vez mais frequentes no Brasil, com estimativa da existência de 10 milhões de pescadores esportivo no país (Freire et al., 2012). De modo geral, os torneios de pesca recreativa no país são organizados em torno de clubes de pesca (principalmente a pesca costeira), que promovem diversos eventos de pesca em todo país (Freire et al., 2017). Esta atividade se beneficia da maior riqueza de espécies de água doce do mundo com 3.124 espécies, e um dos maiores números de espécies marinhas 1.231 espécies (Freire et al., 2014). Além disso, o Brasil possui extenso litoral e importantes bacias hidrográficas que proporcionam as mais diversas experiências da pesca para praticantes de forma recreativa (Freire et al., 2014).

Neste estudo foi possível verificar que nas praias do Olho d'Água e Araçagi houve predominância de espécies das famílias Sciaenidae, Haemulidae e Carangidae. As espécies dessas famílias habitarem águas estuarinas e costeiras, buscando áreas propícias para reprodução e crescimento, considerando que regiões estuarinas além de servirem como habitat permanente para muitas espécies de peixes, possuem papel importante no ciclo de vida de muitas espécies marinhas como área de alimentação, reprodução e proteção contra predadores (Chao, Perreira \& Vieira, 1985; Santana, 2017).

A falta de recursos para realizar estudos que registrem dados dos estoques pesqueiros vem sendo uma preocupação constante da comunidade científica. No sentido de colaborar com as pesquisas, os pescadores esportivos e amadores estão utilizando aplicativos, com objetivo de registrar e monitorar as espécies. Diante dessas estratégias, os pescadores esportivos e amadores se tornam colaboradores em ações de manejo da pesca, atuando no registro de suas capturas e a posterior soltura com foco na sobrevida dos espécimes (Mota, 2019). A ideia de utilizar os aplicativos para auxiliar o monitoramento de dados pesqueiros, visando à produção de informações para criar estratégias de uso sustentável da pesca está sendo discutida amplamente pelos pescadores. Os estoques pesqueiros estão sendo explorados além da conta, em escala mundial, sendo necessário desenvolver e aplicar estratégias sustentáveis de manejo participativo (Mota, 2019), onde todos os envolvidos se tornam peças-chave para a conservação do meio ambiente.

Neste contexto, a prática do pesque e solte é essencial e tem demonstrado bons resultados para manutenção da biodiversidade. A utilização do pesque e solte como medida de gestão não deve ser vista como uma panaceia, aplicável a quaisquer condições (Chaves \& Freire, 2012). É uma prática simples, mas nem por isso dispensa regras e respeito, devendo ser realizada de acordo com a legislação vigente, para que os peixes tenham garantia de sobrevivência ao retornar ao meio ambiente, pois sem o respeito a legislação vigente, o pesque e solte não teria razão de existir como uma alternativa para a sustentabilidade dos recursos naturais (Lopes et al., 2010). 


\section{Referências}

Albano, C. J. \& Vasconcelos, E. C. (2013). Análise de casos de pesca esportiva no Brasil e propostas de gestão ambiental para o setor. Revista Brasileira de Ciências Ambientais, 28, 78-89. Disponível em http://abes-dn.org.br/publicacoes/rbciamb/PDFs/28-10_Materia_8_artigos369.pdf

Almeida, Z. S., Isaac, V. J., Santos, N. B. \& Paz, A. C. (2011) Sustentabilidade dos sistemas de produção pesqueira maranhense. In: M. Haimovici. Sistemas pesqueiros marinhos e estuarinos do Brasil: caracterização e análise da sustentabilidade, pp. 26-40. Rio Grande: Universidade Federal do Rio Grande Furg.

Basaglia, T. P. \& Vieira, J. P. A. (2015). pesca amadora recreativa de caniço na Praia do Cassino, RS: necessidade de informações ecológicas aliada à espécie-alvo. Brazilian Journal of Aquatic Science and Technology, 9(1), 25-29. DOI: 10.14210/bjast.v9n1.p25-29

Beyer, J.E. (1987). On length-weight relationships. Part I: Computing the mean weights of the fish in a given length class. Fishbyte, 5(1), 11-13. Disponível em https://hdl.handle.net/20.500.12348/3367

Brasil. Ministério do Turismo. (2010). Turismo de Pesca: Orientações Básicas. Ministério do Turismo, Secretaria Nacional de Políticas de Turismo. Departamento de Estruturação, Articulação e Ordenamento Turístico, Coordenação Geral de Segmentação. 2 ed. Brasília: Ministério do Turismo. Disponível em http://antigo.turismo.gov.br/sites/default/turismo/o_ministerio/publicacoes/downloads_publicacoes/Turismo _de_Pesca_Versxo_Final_IMPRESSxO_.pdf

Brasil. Lei $\mathrm{n}^{\circ} 11.959$, de 29 de junho de 2009. Dispõe sobre a Política Nacional de Desenvolvimento Sustentável da Aquicultura e Pesca. Brasília. Disponível em https://www.gov.br/agricultura/ptbr/assuntos/aquicultura-e-pesca/legislacao/legislacao-geral-da-pesca/lei-no-11-959-de-29-06-2009.pdf/view

Cardoso, R.L., Cavalcante, A.N., Hetty Salvino Torres, H.S., Nunes, K.B., Ferreira, C.F.C. \& CarvalhoNeta, R.N.F. (2018). Avaliação do crescimento sobre a diversidade de peixes, crustáceos e moluscos nas águas marinhas do estado do Maranhão. Disponível em https://www.scielo.br/j/cab/a/ gWPXdwCtWyJdttcBN9RcnTz/?lang=pt.

Castillo, V. R. A. (1986). Estudo sobre a biologia e o ciclo de vida de Menticirrhus americanus

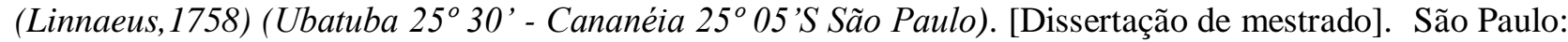
Universidade de São Paulo, Instituto Oceanográfico.

Catella, A. C. (2003). A pesca do Pantanal Sul: situação atual e perspectiva. Corumbá: Embrapa Pantanal. Disponível em https://www.infoteca.cnptia.embrapa.br/infoteca/bitstream/doc/799005/1/DOC48.pdf

Cervigón, F., Cipriani, W. F., Garibaldi, L., Hendrickx, M., Lemus, A. J., Márquez, R., Poutiers, J. M., Robaina, G. \& Rodriquez, B. (1992). Guia de identificación de campo de las espécies comercialess marinas y de águas salobras de la costa septentrional de Sur América. FAO - Food and Agriculture Organization of the United Nations, Roma. 513p. Disponível em http://www.fao.org/3/t0544s/t0544s00.htm

Chaves, P.T. \& Freire, K. M. F. (2012). Pesca esportiva e o pesque e solte: pesquisa recentes e recomendações para estudo no Brasil. Bioikos, 26(1), 29-34. Disponível em http://periodicos.puccampinas.edu.br/seer/index.php/bioikos/article/viewFile/763/743

Chao, N.L, Pereira, L.E. \& Vieira, J.P. (1985) Estuarine fish community of the dos Patos Lagoon, Brazil. A baseline study. In: Yáñez-arancibia, A. (Ed.). Fish community ecology in estuaries and coastal lagoons: towards an ecosystem integration, 429-450. DR(R) Unam, México.

Fernandes, S. C. P., Bentes, A. B., Pereira, L. J. G., Nascimento, M. S. \& Bentes, B. S. (2015). Variação temporal da captura comercial do peixe pedra, Genyatremus luteus, desembarcado em um polo pesqueiro da costa norte do Brasil-Península de Ajuruteua-Bragança-PA. Boletim do Instituto de Pesca, 41(1), 173 - 182.

Félix, F.C., Spach, H.L., Moro, P.S., Schwarz, R. J., Santos, C., Hackradt, C. W \& Silva, M.H. (2007). Utilization patterns of surf zone inhabiting fish from beaches in Southern Brazil, Pan-American Journal of Aquatic Sciences, 2(1), 27-39. 
Figueiredo, M. B., Carvalho-Neta, R. N. F., Nunes, J. L. S. \& Almeida Z. S. (2014). Feeding habits of Macrodon ancylodon (Actinopterygi, Sciaenidae) in northeast, Brasil. Revista Biologia marina y oceanografia, 49, 559-556.

FAO (2020). The State of World Fisheries and Aquaculture 2020. Sustainability in action. Rome. Disponível em https://doi.org/10.4060/ca9229en.

Mello-Filho, E. C. (2020). O relatório 2020 da FAO sobre o estado mundial da pesca e da aquicultura e o objetivo do desenvolvimento sustentável. Disponível: http://www.ibdmar.org/2020/06/o-relatorio-2020-dafao-sobre-o-estado-mundial-da-pesca-e-da-aquicultura-e-o-objetivo-do-desenvolvimento-sustentavel-14-daonu/. Acesso: 27/07/2020.

Freire, K.M.F., Bispo, M.C.S. \& Luz, R.M.C.A. (2014). Competitive marine fishery in the state of Sergipe. Acta of Fisheries and Aquatic Resources, 2(1), 59-72. Disponível em https://seer.ufs.br/index.php/ActaFish/issue/view/145

Freire, K. M. F., Machado, M. L. \& Crepaldi, D. (2012). Overview of inland recreational fisheries in Brazil. Fisheries, 37(11), 484-494. DOI 10.1080/03632415.2012.731867

Freire, K. M. F, Luz, R. M. C. A., Santos, A. C. G., Oliveira, C. S. (2017). Analysis of the enshore competitive recreational fishery in Sergipe. Boletim do Instituto de Pesca, 487-501. DOI 10.20950/16782305.2017

Fricke, R., Eschmeyer, W. N., \& Van Der Laan, R. (2021a). Eschmeyer's Catalog of fishes: genera, species, references. Disponível em http://researcharchive.calacademy.org.

Fricke, R., Eschmeyer, W. N., \& Fong, J. D. (2021b) Species by Family/Subfamily. Disponível em http:// researcharchive.calacademy.org.

Fonteles-Filho, A. A. (2011). Oceanografia, biologia e dinâmica populacional de recursos pesqueiros. Fortaleza: Expressão gráfica editora.

Haluch, C. F. (2008). Aspectos bionômicos da betara Menticirrhus americanus (LINNAEUS, 1758) na Baia de Ubatuba-enseada, São Francisco do sul-SC, Brasil. [Dissertação de Mestrado]. Curitiba.

Haimovici, M., Perreira, S. \& Vieira, P.C. (1996). Distribuição e abundância de teleósteos demersais sobre a plataforma continental do sul do Brasil. Revista Brasileira de Biologia 56(1), 27-50.

ICMBIO, Instituto Chico Mendes de Conservação da Biodiversidade. (2020). Portaria estabelece regras para pesca esportiva em UCs. Disponível em https://www.icmbio.gov.br/portal/ultimas-noticias/20geral/10874-portaria-estabelece-regras-para-pesca-esportiva-em-ucs.

Ivo, C.T.C. \& Fontelles-Filho, A. A. (1997). Estatística pesqueira: Aplicação em Engenharia de Pesca. Fortaleza: Tom Gráfica e Editora.

Lopes, P. R. D., Silva, J. T. O. \& Fernandes, I. T. (2010). Nota prévia sobre alimentação de Conodon nobilis (LINNAEUS, 1758) (Actinopterygii: Haemulidae) na praia de Malhado, Ilhéus (Bahia). In Simpósio de Biologia Marinha, 13, São Paulo.

Magro, M.; Cergole, M.C. \& Rossiwongtschowski, C.L.D.B. (2000). Avaliação do potencial sustentável de recursos vivos na Zona Econômica Exclusiva - Revizee - Síntese de conhecimentos dos principais recursos pesqueiros costeiros potencialmente explotáveis na costa Sudeste-Sul do Brasil: Peixes. Ministério do Meio Ambiente, CIMP - Comissão Interministerial para os Recursos do Mar, São Paulo, 154p.

Mclachlan, A. (1980). Exposed Sandy beaches as semi- closed ecosystems. Marine Environmental Research, 4, 59-63. DOI:10.1016/0141-1136(80)90059-8

Menezes, N. A. \& Figueiredo, J. L. (1980). Manual de peixes marinhos do Sudeste do Brasil. IV. Teleostei (3). São Paulo: Museu de Zoologia da Universidade de São Paulo. 
Menezes, N. A., Buckup, P. A., Figueiredo, J. L. \& Moura, R. L. (2003). Catálogo das espécies de peixes marinhos do Brasil. São Paulo: Museu de Zoologia da Universidade de São Paulo.

MPA - Ministério Da Pesca E Aquicultura. (2012). Instrução normativa $n^{\circ} 5$ de 13 de junho de 2012. Brasília.

Mota, A. (2019). Aplicativo para registro e monitoramento de peixes é lançado. Fish TV. Disponível em https://www.fishtv.com/noticias/geral/aplicativo-para-registro-e-monitoramento-de-peixes-e-lancado?fbclid =IwAR3czX8rjPIzcNq-Kcetx7S_bNrC48ugjvQojFIrK78UfClfhW8cfDeOfNw.

Mulato, I. P. Corrêa, B. \& Vianna, M. (2015). Distribuição espaço temporal de Micropogonias furnieri (PERCIFORMES, SCIAENIDAE) em um estuário tropical no sudeste do Brasil. Boletim do Instituto de Pesca, 41(1), 1-18. Disponível em https://www.pesca.sp.gov.br/41_1_1-18.pdf

Nunes, Y. B. S., Aranha, M. B., Freitas, J., Fernandes, J. F. F., Silva, L. R., Figueiredo, M. B. (2020). Length at first sexual maturity of economically important fishes in the Brazilian Northeast Coast. Ocean and Coastal Research, 68, 1-5. DOI: 10.1590/S2675-28242020068311

Pitcher, T. J. (1999). Evaluating the Benefits of Recreational Fishing: Papers, Discussions and Issues: a Conference Held at the UBC Fisheries Centre, June 1999. Fisheries Centre.

Rodrigues, E. A. (2013). Avaliação dos Resíduos gerados no processo produtivo de pescado na colônia de pescadores z3, Pelotas- $R S$ produtivo de pescado na colônia. 0-58.

Rondineli, G. R.; Braga, F. M. S.; Tutui, S. L. S. \& Bastos, G. C. C. (2007). Dieta de Menticirrhus americanos (LINNAEUS, 1758) e Cynoscion jamaicensis (VAILLANTE AND BOCOURT,1883) (PISCES, SCIAENIDAE) no sudeste do Brasil, Estado de São Paulo. Boletim do Instituto de Pesca de São Paulo, 33(2), 221 - 228. Disponível em https://www.pesca.sp.gov.br/33_2_221-228.pdf

Santana, T.C., Carvalho-Neta, R.N.F., Fernandes, J.F.F., Lobato, R.S., Castro, J.S., Castro, J.J.P., Barbosa, J.M. \& Teixeira, E.G. (2019). An Illustrated Guide to Commercial Teleost Fishes from Upaon-Açu Island, Brazil. Editora UEMA. Disponível em http://www.ppgrap.uema.br/wp-content/uploads/2019/07/Santana-etal.-2019-An-Illustrated-Guide-To-Commercial-Teleost-Fishes-From-Upaon-A\%C3\% A7u-IslandS\%C3\%A3o-Lu\%C3\%ADs-Island2.pdf

Santana. T. C. 2017. Morfologia e taxonomia dos peixes (Actinopterygii: Teleostei) marinhos e estuarinos comerciais do Maranhão. [Dissertação de mestrado]. São Luís: Universidade Estadual do Maranhão.

Santana, T.C., Teixeira, E.G., Castro, J.S., Fernandes, J.F.F, Lobato, R.S. \& Carreiro, C.R.P. (2016). Principais espécies de Sciaenidae marinhas e estuarinas comercializadas na Ilha do Maranhão. Em Zafira da Silva de Almeida, Raimunda Nonata Fortes Carvalho Neta, Débora Martins Silva Santos (Eds.) Recursos Pesqueiros e Pesca Artesanal no Maranhão: Sumário Executivo (pp. 41-59). São Luís: Universidade Estadual do Maranhão.

Santos, N. B. (2007). Biologia reprodutiva de peixes Cianídeos capturados nas proximidades dos terminais portuários do Pará e Maranhão. [Dissertação de mestrado]. Belém: Universidade Federal do Pará.

Silva-Júnior, C. A. B, Lira, A. S., Eduardo, L. N., Viana, A. P., Lucena-Frédou, F. \& Frédou, T. (2019). Ichthyofauna bycatch of the artisanal fishery of penaeid shrimps in Pernambuco, northeastern Brazil. Boletim do Instituto de Pesca, 45(1), 1-10. DOI: 10.20950/1678-2305.2019.45.1.435

Lira, A. S., Eduardo, L. N., Viana, A. P., Lucena-Frédou, F. \& Frédou, T. (2019). Population structure, size at first sexual maturity, and feeding ecology of Conodon nobilis (Actinopterygii: perciformes: haemulidae) from the coasts of Pernambuco, north-eastern Brazil. Acta Ichthyologica et Piscatoria, 49(4), 389-398. DOI:10.3750/AIEP/02578

Lewis, D. D. S., Braun, A. S. \& Fontoura, N. F. (2001). Relative seasonal fish abundance caught by recreational fishery on Cidreira Pier, southern Brazil. Journal Applied Ichthyology, Kiel, 15, 149-151. DOI: 10.1046/j.1439-0426.1999.00134.x 
Souza, R. A. O. (2016). Análise ecomorfológica da família Carangidae residente na zona de arrebentação de praias na Baía de Santos. Congresso Nacional de Iniciação Cientifica, (Sindicato das Mantenedoras de Ensino Superior) São Paulo.

Vazzoler, G. (1975). Distribuição da fauna de peixes demersais e ecologia dos Sciaenidae da plataforma continental brasileira, entre as latitudes $29^{\circ} 21^{\prime} \mathrm{S}$ (Tôrres) e $33^{\circ} 41^{\prime} \mathrm{S}$ (Chuí). Boletim do Instituto Oceanográfico, 24, 85-169.

Vazzoler, A. E. A. M. (1991). Síntese de conhecimento sobre a biologia da corvina, Micropogonias furnieri (Desmarest, 1823), da costa do Brasil. Atlantica, 13(1), 55-75.

Vazzoler, A. E. A. M. (1996). Biologia da reprodução de peixes teleósteos: teoria e prática. Maringá: Eduem/ CNPq/ Nupelia.

Vilar, CC., Joyeux, J.C., Giarrizzo, T., Spach, H.L., Vieira, J.P. \& Vaske-junior, T. (2013). Local and regional ecological drivers of fish assemblages in Brazilian estuaries. Marine Ecology Progress Series, 485, 181-197. DOI: $10.3354 / \mathrm{meps} 10343$

Zagonel, L. B. (2006). Testando a monofilia de Pachyurinae (Perciformes, Scianidae) e de seus componentes com base em dois genes mitocondriais. [Monografia de graduação] Curitiba, Universidade Federal do Paraná, Departamento de Zoologia.

Zar, J. H. (2010). Biostatistical Analysis. 5th Edition, Prentice-Hall/Pearson, Upper Saddle River, xiii, 944. 